\title{
Structural Analysis of Macromolecule-Cobalt Nanoparticle Complexes
}

\author{
M.A. Zalich, ${ }^{* * * *}$ M. Saunders, ${ }^{* * * V}$ V.V. Baranauskas, ${ }^{* M}$ M.L. Vadala, ${ }^{* J . S . ~ R i f f l e * ~ a n d ~ T . G . ~ S t . ~ P i e r r e * * ~}$
}

* Department of Chemistry and Macromolecules and Interfaces Institute, Virginia Polytechnic Institute and State University, Blacksburg, VA 24060, USA

** School of Physics, The University of Western Australia, Crawley, WA 6009, Australia

*** Center for Microscopy and Microanalysis, The University of Western Australia, Crawley, WA 6009, Australia

Magnetic nanoparticles have been under intense investigation due to their potential applications in the biomedical industry. Some of these applications include MRI contrast agents, tumor hyperthermia, biotoxin removal and gene/drug delivery. Most research has been conducted on nanoparticles of various iron oxides, $\mathrm{Ni}, \mathrm{Co}$ and $\mathrm{Fe}$. Cobalt has one of the largest magnetic susceptibilities of these materials, but it readily oxidizes upon exposure to air to afford the antiferromagnetic oxide. Protecting Co against oxidation is crucial to its use in biomedical applications and the structural analysis of oxidatively stable nanoscale macromolecule/cobalt complexes will be discussed herein.

Macromolecule-cobalt complexes were prepared with two dispersant systems: 1) poly(styrene- $b-4-$ vinylphenoxyphthalonitrile) (S1) [1] and 2) poly(dimethylsiloxane- $b$-(methylvinylsiloxane-comethyl(2-trimethoxysilethyl)siloxane)) (S2) [2]. Dried nanoparticle complexes were thermally treated at temperatures between $600-700^{\circ} \mathrm{C}$ to generate graphite or silica sheaths around the particles. Structural analysis of the macromolecule/cobalt nanomaterials before and after heat treatment was conducted on a JEOL $3000 \mathrm{~F}$ field-emission transmission electron microscope operated at $300 \mathrm{kV}$ and equipped with a Gatan image filter. X-ray diffraction was performed using a Siemens D-500 x-ray diffractometer $\left(\mathrm{Cu}-\mathrm{K} \alpha\right.$ radiation) from $2 \theta=10-110^{\circ}$.

Microscopic analysis of S1 indicated an increase in particle size distribution from the pre-heattreated sample (range of 9 to $45 \mathrm{~nm}$ ) to the heat-treated sample (range of 7 to $147 \mathrm{~nm}$ ), which indicated the occurrence of cobalt nanoparticle sintering. Co distribution maps of the pre-heattreated and heat-treated samples, acquired using energy filtered TEM, correlated well with the corresponding bright-field images and confirmed the elemental identity of the nanoparticles. Highresolution TEM, selected area electron diffraction (SAD) and nano-beam electron diffraction (NBD) were used to elucidate crystallographic information about the pre-heat-treated and heat-treated samples. The pre-heat-treated sample was weakly crystalline, which led to diffuse diffraction patterns. HRTEM of the heat-treated sample showed multilayer coatings around crystalline particles, which had spacings consistent with the interlayer spacings of graphite [3]. Some HRTEM analyses indicated that hcp-cobalt was present; however, NBD of a crystalline particle revealed the [011] axis of fcc-cobalt (Figure 1) [4]. XRD revealed the dominant phase of both the pre-heattreated and heat-treated systems to be fcc with other phases of cobalt possibly present. Magnetic measurements showed that the heat-treated sample is protected against oxidation for over one year.

Particle size analysis of S2 indicated an increase in particle size distribution from the pre-heattreated sample (range of 8 to $38 \mathrm{~nm}$ ) to a sample thermally treated at $600^{\circ} \mathrm{C}$ (range of 4 to $750 \mathrm{~nm}$ ). Thermal treatment at $700^{\circ} \mathrm{C}$, however, resulted in a particle size distribution ranging from 4 to 21 
$\mathrm{nm}$. The pyrolysis of the polymer may be slower at $600^{\circ} \mathrm{C}$ enabling the cobalt nanoparticles to come into contact with one another and sinter, while pyrolysis at $700^{\circ} \mathrm{C}$ may lock the cobalt nanoparticles in place within the silica matrix thus preventing sintering. HRTEM and electron diffraction studies were conducted on the pre-heat-treated and heat-treated samples. The pre-heat-treated sample was weakly crystalline affording diffuse diffraction patterns. The two heat-treated samples were highly crystalline, with the resulting HRTEM images and diffraction patterns indicating complex crystalline structures (Figure 2). XRD provided conclusive data that the dominant phase in the heat-treated samples was fcc, while no conclusive data were obtained for the pre-heat treated sample. Magnetic measurements revealed a detectable amount of cobalt oxide in the thermally treated samples.

Oxidatively stable cobalt nanoparticles were prepared by thermally treating $\mathrm{S} 1$ /cobalt complexes to afford graphitic, oxygen impermeable coatings around the particles. The thermal treatment procedure used for the S2/cobalt nanoparticle system needs improvement to decrease oxygen permeability. The dominant crystal phase for all systems is fcc-cobalt; however, other phases may be present as indicated by HRTEM and electron diffraction data. Further studies are in progress to control the particle size during thermal treatment and to functionalize the surfaces of thermally treated particles rendering them dispersible in various media $[5,6]$.

\section{References}

[1] V.V. Baranauskas, M.A. Zalich et al., Chem. Mater. (2004) Submitted.

[2] M.L. Vadala et al., Polymer, 49 (2004) 7445.

[3] C.H. Kiang et al., Phys. Rev. Lett., 81 (1998) 1869.

[4] D.B. Williams et al., In Transmission Electron Microscopy; Plenum Press: New York, 1996.

[5] M.L. Vadala, M.A. Zalich et al., J. Magn. Magn. Mater. (2004) Submitted.

[6] Research supported by: the Australian-American Fulbright Commission and DARPA-AFOSR.
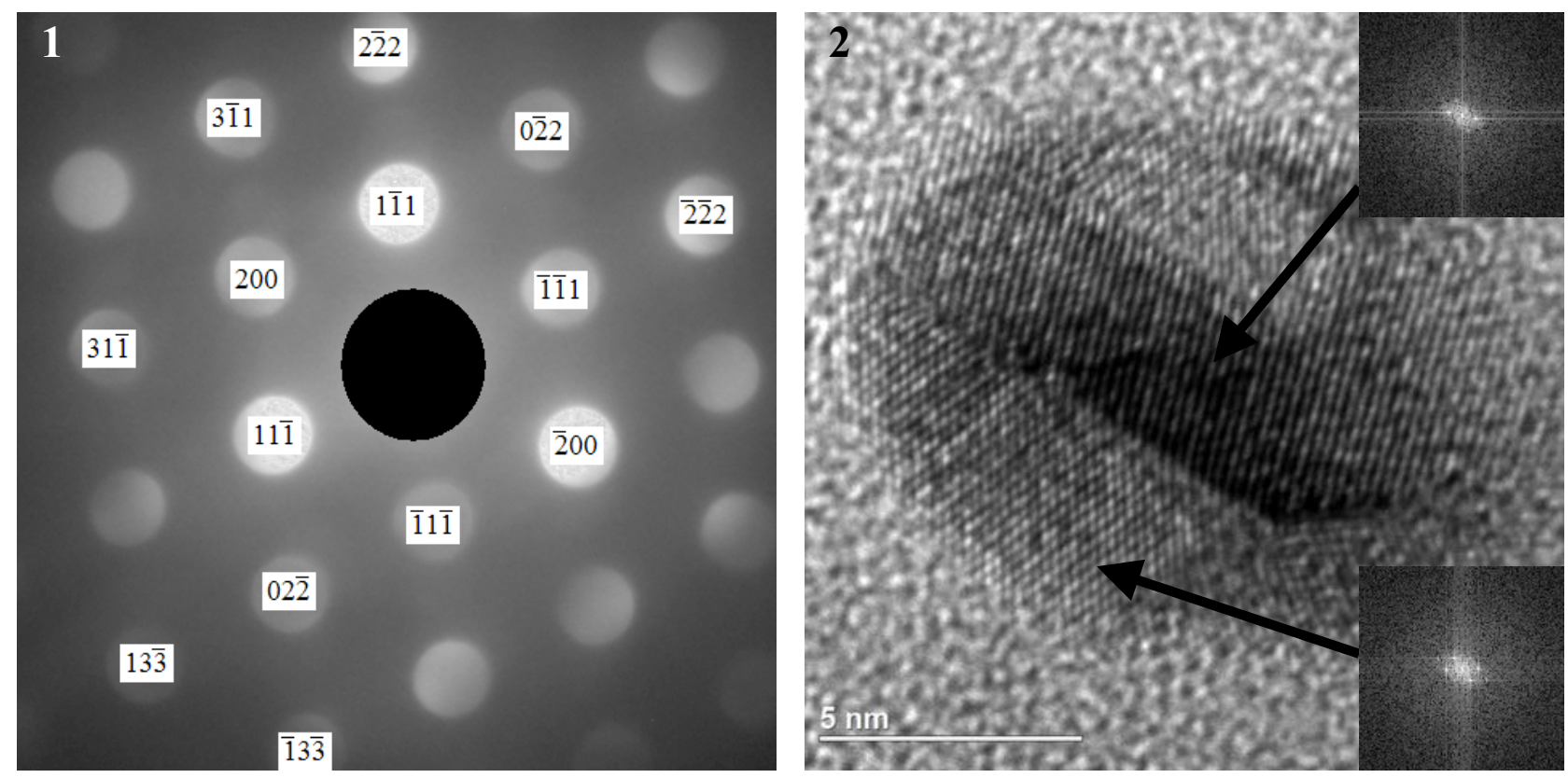

Figure 1: Nano-beam diffraction pattern of [011] zone axis of fcc cobalt.

Figure 2: HRTEM image of multi-twinned cobalt nanocrystal heated at $700^{\circ} \mathrm{C}$. Insets are Fourier transforms of corresponding twin planes. 